\title{
Digital workflow for the accurate computation of the geometric properties of bamboo culms for structural applications
}

\author{
Rodolfo Lorenzo, ${ }^{1, *}$, and Leonel Mimendi ${ }^{1}$ \\ ${ }^{1}$ Department of Civil, Environmental and Geomatic Engineering, University College London, London, UK
}

\begin{abstract}
Bamboo is one of the most promising sustainable construction materials due to the large endemic natural reserves prevalent in the Southern Hemisphere. However, industrialised materials, such as concrete, steel and aluminium have overshadowed the application of natural bamboo culms, due to the high-quality assurance achieved over decades refining the production processes of structural elements manufactured from the former. As a result, the physical, geometric and mechanical properties of these industrialised structural elements are quantifiable, predictable and in agreement with international standards. This research presents the details of a digital workflow to quantify the inherent geometric variability of bamboo culms as part of a new quality assurance process for this natural structural element. This workflow relies on the use of a mid-range, commercially available structured-light 3D scanner to accurately capture a point cloud of the bamboo geometry and generate a corresponding polygon mesh. Digital models of three different bamboo species were validated through comparison with key physical measurements finding that the adoption of these digital models can significantly improve the accuracy and efficiency of manual methods due to the complex irregularities found in bamboo culms. This work demonstrates the benefits of adopting a non-destructive, reverse-engineering approach to quantify the geometric properties of bamboo compared to traditional tools and methods. Overall, this research shows the potential of digital technologies to support the adoption of this natural material allowing for the re-assessment of design workflows and providing an opportunity for bamboo to compete with industrialised materials.
\end{abstract}

\section{Introduction}

\subsection{Natural geometry}

Bamboo is one of the most promising sustainable construction materials due to the large endemic natural reserves prevalent in the Southern Hemisphere. In its natural state (round pole) bamboo has been mainly used for construction purposes. Processed bamboo (bamboo strips or fibres used for composites) has been traditionally used to fabricate a wide and ever-growing list of products like woven panels, home accessories and furniture, among others. However, industrialised materials, such as concrete, steel and aluminium have overshadowed the application of natural bamboo culms due to the high-quality assurance achieved over decades refining the production processes of structural elements manufactured from the former. As a result, the physical, geometric and mechanical properties of these elements are quantifiable, predictable and in agreement with international building standards. The wood industry has also experienced a stronger development compared to bamboo. Despite wood being a natural material, the elements that are commonly used for construction purposes are cut down into uniform, prismatic and standard shapes and sizes (e.g. BS EN 336:2013). This approach helps to reduce the uncertainty of geometric properties and allows a more reliable use of the material.

Bamboo can be described as a long tapered hollow cylinder, with intermittent transverse membranes along its axis, known as "nodes". From all the different species of bamboo, only some of them are regarded as suitable for structural purposes [1]. The internal biological composition of bamboo is well known [1,2], as well as its fast-growing properties [3]. Bamboo has proven to have a huge potential as a substitute for wood (and other industrialised materials) in a wide range of uses, one of which is in structural applications. Moreover, bamboo can be produced in rural areas, 
enhancing economic growth and reducing the evergrowing pressure over urbanisation [4].

This research presents the details of a digital workflow to quantify the inherent geometric variability of bamboo culms as a new quality assurance process for this natural structural element. For over five decades, studies on bamboo poles have focused on its mechanical, physical and biological properties, but there has been little focus on its geometrical variability, its significance on fabrication quality and its mechanical behaviour [5]. Janssen [6] compared a bamboo pole with similar industrialised structural elements, such as concrete or steel. He found that the main geometric variabilities on the geometry of bamboo were due to a) tapering, b) irregular internodal distances, c) variable cross sectional properties along its axis and d) out-of-straightness. As such, the accurate determination of geometric properties is fundamental to study the mechanical properties of round bamboo [7-11], behaviour of connections [12,13] and structural analysis $[14,15]$ for which determining the pole's geometric variability is fundamental. Nevertheless, the only official international standard, ISO 22157-1-2004 [16], focused on the determination of the physical and mechanical properties of bamboo, is based on basic measurements of a limited and discrete number of properties that fail to capture the significant variation in the geometry of bamboo poles described by Janssen [6].

During the last two decades there have been studies that measured and analysed the variability of the geometric properties in bamboo poles. Amada et al [2] and Chung \& Yu [7] performed similar studies to determine the variability of diameter, thickness and section properties (area and second moment of area) along the length of the bamboo, however they did not describe the measurement methodology adopted. A study by Ghavami \& Moreira [5] focused on the influence of geometric imperfections, where they designed a non-destructive, mapping equipment to find the maximum initial imperfection of the element (outof-straightness) as well as the cross-section variability along the culm. The geometric properties were used to calculate mechanical properties which resulted in good agreement when compared with mechanical tests. A simpler method was then designed by Richard [8] who also considered the effect of the out-of-straightness of the bamboo axis on its capacity. A more recent work on bamboo structures [17] also included the impact of the geometric effects on their study. ISO 22157-1-2004 [16] was used to measure the diameter and thickness in different sections of the culm, as well as the method described by Richard [8] to extract out-of-straightness. Additionally, a method to calculate the eccentricity for each cross-section was applied, defined as the deviation of a conic section from a perfect circle measured at both principal axes.

Due to the complexity of the aforementioned methods, a challenge remains to ensure the reliability and repeatability of the results obtained through practical implementation, considering the significant variability of bamboo poles. Therefore, the importance of obtaining accurate and reliable measurements is undeniable. A study [18] pointed out that there are five aspects on which the geometric variability of bamboo has great impact: compression behaviour, flexural behaviour, grid-shells (or any 3D structure), visual grading and structural classification. However, manual measuring tools, adequate for most industrialised structural elements, are not necessarily the most suitable solution to address the challenge of accurately determining the geometric properties of bamboo poles. This research is therefore focused on adopting new non-contact, non-destructive, reverse-engineering technologies to overcome this challenge [19].

\subsection{Bamboo digitisation}

A 3D model that truly represents the natural shape of a bamboo pole can enable the study of the different geometric parameters of the pole, thus quantify its geometric variability. A three-dimensional (3D) model is a virtual representation of an object's geometry. The model can be manually built in a Computer-Aided Design (CAD) software if the geometry of the object is known. For "uniform", manufactured objects, the determination of their geometry is a straightforward process. Columns and beams in civil engineering or shafts and bearings in mechanical engineering are a good example of uniform objects. The virtual reconstruction of "non-uniform" natural objects however, is considerably more intricate and complex because of their more complicated irregular geometry.

For centuries, manual measurements have been the only way to acquire the geometry of objects, nevertheless, their accuracy has been questionable when applied on complex objects. Non-intrusive methods have been developed to acquire the geometry of objects [20], but only in the last two decades, with the emergence of digital technology, new imaging methods have been developed to acquire and build virtual models. The main methodologies involved the use of photographs [21,22], video-recording [23], laser sensors $[24,25]$ and LED light projections [26,27].

Photogrammetry is a methodology that is normally used when the object can be represented by line-based structures, especially if the object has distinct texture. Laser and light scanner techniques are more useful when irregular objects like sculptures, reliefs or archaeological sites are involved [28]. More recently, it has been proved $[29,30]$ that a combination of both methods can also be applied into large (e.g. buildings) and small (e.g. coins) objects to acquire both, high accuracy representations of non-uniform objects and their real colour.

This workflow relies on the use of a mid-range, commercially available structured-light 3D scanner [19] to accurately capture the bamboo geometry from which 
a point cloud model is generated. This point cloud is post-processed to be merged in to triangular polygons that compose the virtual surface of the bamboo. In contrast with other structural elements, bamboo poles are not suitable for geometric standardisation as each geometric parameter can display significant variability due to its nature [31]. Thus, the formal adoption of bamboo poles in structural applications requires this variability to be efficiently and accurately captured treating each individual pole as a ready-to-use structural element.

\section{Materials and methodology}

\subsection{Material description}

The geometric properties of bamboo poles will vary not only among species but also along single elements [31] and thus three different species from three different global locations were adopted for this study (Table 1). All bamboo poles were randomly selected to achieve a broad distribution of physical and geometric parameters (surface imperfections, curvature, nodal spacing, etc) to reflect the natural features of each specie.

\subsection{Scan methodology}

\subsubsection{Scan subject}

From a 3D scanning perspective, a bamboo pole can be described as a cylindrical slender element with a very small depth-to-span ratio. The internode surface is smooth and relatively shiny, with no distinctive texture or changes in geometry. The intermediate nodes along its length exhibit characteristic ridges and indentations (cut-off branch growths) with differing patterns and an increase in average diameter compared to the adjacent internodes. Oldhamii and Guadua bamboo presented similar straw colour, with shaded brown or light yellow small areas randomly sparse along the pole. Some Guadua presented small black irregular patches in random locations which are typical of the species. Moso bamboo had a dark olive colour due to the local treatment of carbonisation. The ends of all poles were cut at the internode to allow an unrestricted view of the pole wall and a portion of its inner surface.

\subsubsection{Scan system}

The equipment selected for the scanning system was an Artec Eva scanner [32] which is a hand-held device that operates based on structured light sensor technology [33]. Artec Eva has a resolution of up to 0.5 $\mathrm{mm}$ and a $3 \mathrm{D}$ point accuracy of $0.1 \mathrm{~mm}$. The texture resolution is $1.3 \mathrm{mp}$ and it supports $24 \mathrm{bpp}$ of colour. It projects a white LED structure light using flash bulbs with a maximum frame rate of $16 \mathrm{fps}$ and an exposure time per frame of $0.0002 \mathrm{~s}$. The scanning range of the device is 0.4 to $1.0 \mathrm{~m}$ with a linear field of view of 214 x $148 \mathrm{~mm}$ to $536 \mathrm{x} 371 \mathrm{~mm}$ (height $\mathrm{x}$ width) respectively. The scanner is able to acquire a maximum of 2 million points per second (point cloud).

The scanner was operated using a laptop Dell XPS 15 equipped with an Intel i7-6700HQ CPU @ 2.66 $\mathrm{GHz}, 16 \mathrm{~GB}$ of installed memory and a dedicated video card Nvidia GTX GeForce $960 \mathrm{~m}$ with 4 GB of memory. Processing of the point cloud was carried out using Artec's proprietary software Artec Studio 12 [32] in a work station Dell Precision with an Intel Xeon E51620v3 CPU@3.5 GHz, 32 GB of memory and a dedicated video card Nvidia Quadro K2200 with 4 GB of memory.

\subsubsection{Scanner parameters configuration}

The scanning process was configured specifically to optimise the acquisition of the bamboo geometry. The key parameters involved in the development of the process were: scanning time, file size and point cloud processing time. The parameters and the equations for the optimal geometry acquisition were obtained through experimental tests using the scanner, a range of bamboo sizes and variations of the set-up. The final optimal details, are described as follows.

The number of frames $\left(\mathrm{F}_{\mathrm{n}}\right)$ acquired was crucial for the scanning process. This parameter depends on the scanning time $\left(\mathrm{S}_{\mathrm{t}}\right)$ in seconds, and the acquisition rate expressed in frames per second (fps):

$$
\begin{gathered}
F_{n}=120+\left(S_{t} * f p s\right) \\
S_{t}=\frac{L}{T_{s}} \\
T_{s}=65 * r p s
\end{gathered}
$$

Where $T_{s}$ is the translation speed of the bamboo along its length $(\mathrm{mm} / \mathrm{s}), \mathrm{L}$ is the total length of the pole (m), 65 is the translation in millimetres per rotation and 120 was the average number of frames required to acquire the inner bamboo surface at each end. The scan path consisted of simultaneous rotation and translation along the bamboo's axis, therefore, a minimum circumferential and longitudinal overlapping was needed. Figure 1 demonstrates the overlapping generated while scanning. The yellow frame (set of points) was the first shot taken by the scanner. As the pole simultaneously rotates and translates, the scanner will take a second shot, represented by the blue frame. The scanner continues taking shots until the pole rotates $360^{\circ}$ and translates $0.065 \mathrm{~m}$. This is considered a full cycle and is represented by the green frame. The helical scan will allow every frame to have both, a circumferential and a longitudinal overlapping. To geometrically ensure this, the translation speed will then depend on the revolutions per second (rps) that the bamboo is rotated at:

$$
r p s=\frac{f p s}{F_{r}}
$$


Table 1. General description of materials

\begin{tabular}{cccccccc} 
Bamboo Specie & Origin & $\begin{array}{c}\text { No. of } \\
\text { poles }\end{array}$ & $\begin{array}{c}\text { Age } \\
\text { (years) }\end{array}$ & $\begin{array}{c}\text { Length } \\
\text { (m) }\end{array}$ & $\begin{array}{c}\text { Diameter } \\
\text { (mm) }\end{array}$ & Treatment & Working site \\
\hline $\begin{array}{c}\text { Moso (Phylostachys } \\
\text { Pubescens) }\end{array}$ & $\begin{array}{c}\text { Jiangsu, P.R. } \\
\text { China }\end{array}$ & 5 & 3 to 4 & 3.30 & 85 & $\begin{array}{c}\text { Carbonization/ } \\
\text { Env. Chamber }\end{array}$ & $\begin{array}{c}\text { Nanjing Forestry } \\
\text { University, China }\end{array}$ \\
$\begin{array}{c}\text { Oldhamii (Bambusa } \\
\text { Oldhamii) }\end{array}$ & $\begin{array}{c}\text { Veracruz, } \\
\text { Mexico }\end{array}$ & 10 & 3 to 5 & 5.00 & 65 & $\begin{array}{c}\text { Leaching/ } \\
\text { Air-dried }\end{array}$ & UNAM, \\
Muadua (Guadua & $\begin{array}{c}\text { Valle del Cauca, } \\
\text { Colombia }\end{array}$ & 10 & 2 to 5 & 3.00 & 110 & $\begin{array}{c}\text { Leaching/ } \\
\text { Air-dried }\end{array}$ & UCL, United \\
Angustifolia Kunth) & Kingdom
\end{tabular}

Table 2. Scanning time and number of frames estimation

\begin{tabular}{c|cccccccccccccc}
\multicolumn{2}{c}{} & Length (m) & 0.5 & 1 & 1.5 & 2 & 2.5 & 3 & 3.5 & 4 & 4.5 & 5 & 5.5 & 6 \\
\hline $\begin{array}{c}\mathbf{f p s}=\mathbf{8} \\
\mathbf{T s}=\mathbf{1 7} \mathbf{~ m m} / \mathbf{s}\end{array}$ & Scanning time (min) & 0.5 & 1.0 & 1.4 & 1.9 & 2.4 & 2.9 & 3.4 & 3.8 & 4.3 & 4.8 & 5.3 & 5.8 \\
& Frame Number & 355 & 591 & 826 & 1061 & 1296 & 1532 & 1767 & 2002 & 2238 & 2473 & 2708 & 2944 \\
\hline $\begin{array}{c}\mathbf{f p s}=\mathbf{4} \\
\mathbf{T s}=\mathbf{9} \mathbf{~ m m} / \mathbf{s}\end{array}$ & Scanning time (min) & 1.0 & 1.9 & 2.9 & 3.8 & 4.8 & 5.8 & 6.7 & 7.7 & 8.7 & 9.6 & 10.6 & 11.5 \\
& Frame Number & 342 & 564 & 787 & 1009 & 1231 & 1453 & 1676 & 1898 & 2120 & 2342 & 2564 & 2787
\end{tabular}

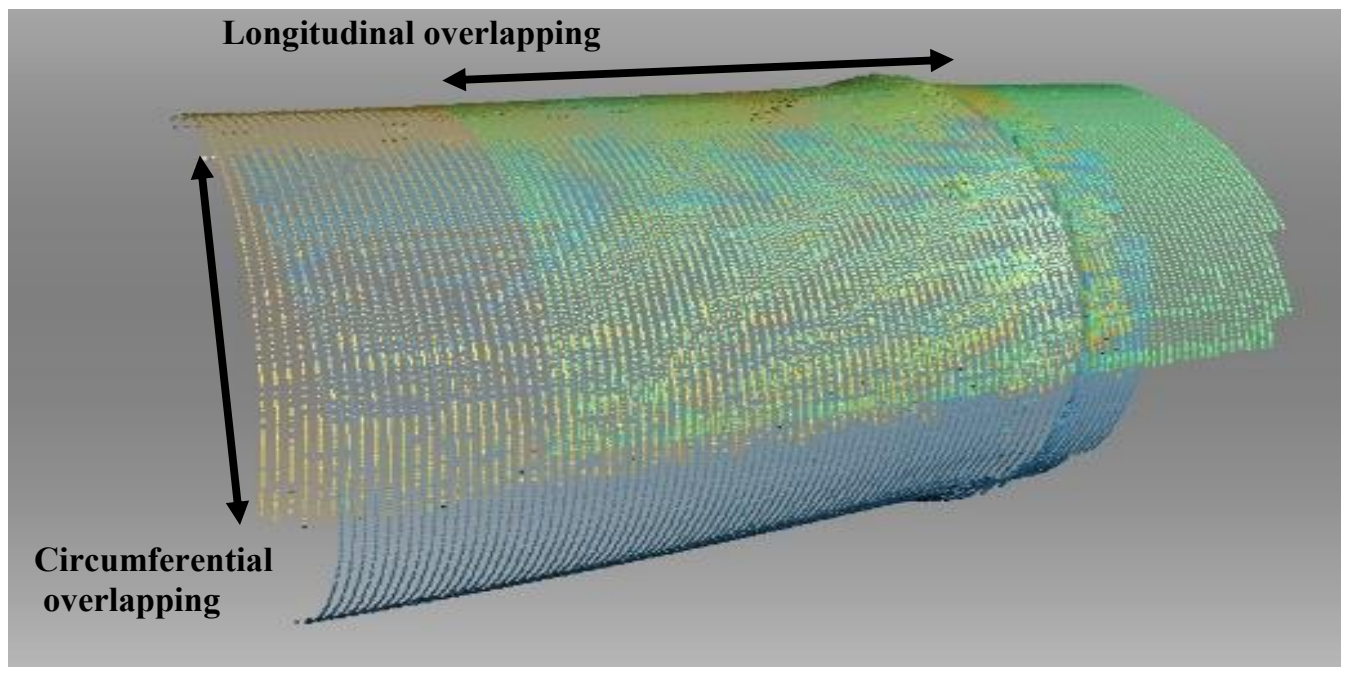

Figure 1. Circumferential and longitudinal overlapping

Where $F_{r}$ is the number of frames needed per cycle of rotation. Through an iterative process the number of frames per rotation to efficiently scan poles of $60 \mathrm{~mm}$ to $150 \mathrm{~mm}$ diameter range was found to be 30 frames. Based on Equation 2, the scanning time (Table 2) was calculated for different lengths and translation speeds. File size and point cloud processing time also depends on the number of frames acquired. Figure 2 and 3 show the relationship of both parameters with frame number.
Figure 2 depicts a linear incremental relationship of file size as the frame number increases, however, in Figure 3, it is noticeable that the processing time of

point cloud increments exponentially with the number of frames. The size is directly related with file management operations (e.g. saving, copying, etc.), whereas the processing of the point cloud itself consists of the registration of the points acquired and generation of a mesh file.

In summary, the frame number was kept to the lowest possible value to optimise the total time of the 
scanning procedure. Table 3 shows the final configuration parameters of the adopted procedure.

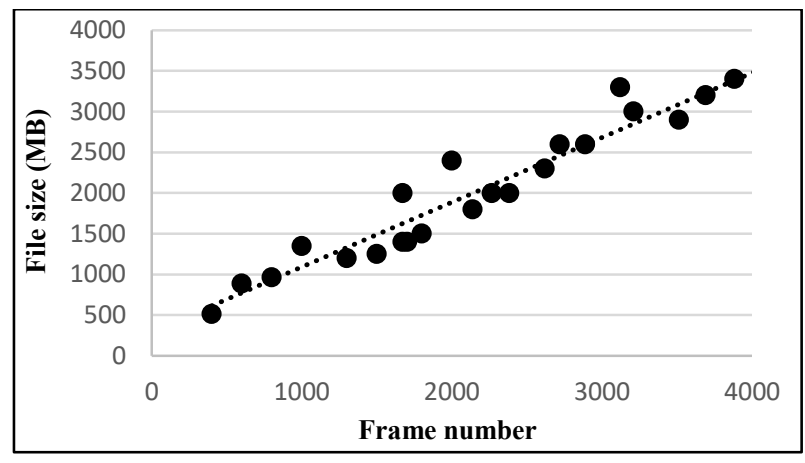

Figure 2. File size per number of frames

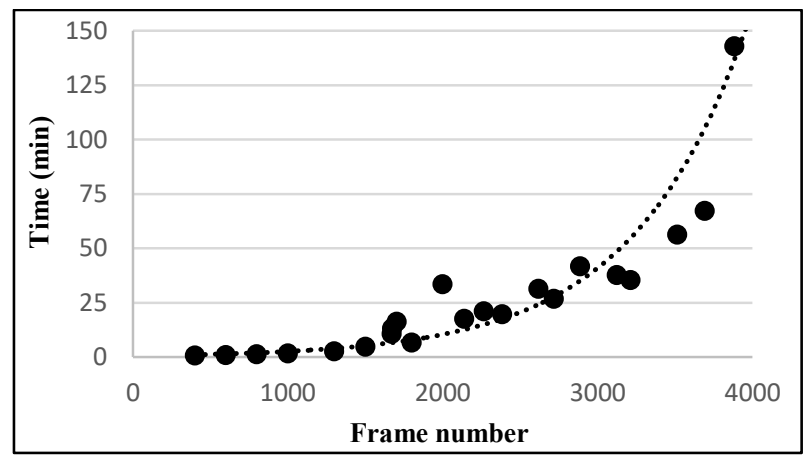

Figure 3. Point cloud processing time per number of frames

Table 3. Parameters adopted for scanning procedure

$\begin{array}{lc}\text { Geometry } & \text { Yes } \\ \text { Texture } & \text { Yes } \\ \text { Scanner-to-object distance (mm) } & 700 \\ \text { Field of view @700 mm (height x width - mm) } & 375 \times 260 \\ \text { Frames per second (fps) } & 8 \\ \text { Maximum length of pole (m) } & 4 \\ \text { Maximum number of frames } & 2000 \\ \text { Maximum scan time (min) } & 4 \\ \text { Maximum File size (GB) } & 2 \\ \text { Maximum processing time (min) } & 10\end{array}$

\subsubsection{Scanner set-up and process}

The scanning set-up was developed considering simplicity and portability for on-site use. Scanner and bamboo motions were limited to reduce the effect of human factors during operation, which can affect the quality of the acquisition process. The workflow also considered that a single scan session was required to reduce noise and errors in the points acquired and therefore, increase the efficiency of the processing time [34]. The general set-up diagram is shown in Figure 4.

The scanning set-up consisted of four pipe stands, equipped with ball heads set at an angle of $120^{\circ}$ (Figure 5), to support bamboo poles with diameter between $60 \mathrm{~mm}$ and $150 \mathrm{~mm}$ at four equally spaced points along their length (Figure 4). The scanner was mounted on a camera dolly to follow a semi-circular path of $700 \mathrm{~mm}$ radius on a workbench (Figure 4). The height of the pipe stands at both ends was set $20 \mathrm{~mm}$ lower than the inner two, allowing a smooth translation as the bamboo pole moves along the supports.

Prior to scanning, two scanning target references were positioned at the bottom end of the pole and a single target at the top. These targets were used as a physical reference for the digital model and consisted of M5 socket head button screws inserted in predrilled holes so that their $7 \mathrm{~mm}$ diameter hemispherical head is used as the scanned target (Figure 6). The position of these targets was within one diameter away from the end nodes.

The starting point of the scanning workflow is shown in Figure 4, with the scanner and bamboo positioned in a way that the bamboo bottom end and a portion of its inner surface were captured while the pole was rotated one full revolution around its axis. The scanner was subsequently slid $45^{\circ}$ to the central position so that the bamboo could be scanned during a simultaneous rotational motion around its own axis and translation along its length. This motion creates a helical scan that must overlap throughout the entire bamboo surface acquisition (Figure 1). After the bamboo top end had reached the scanner central position, the scanner was slid $45^{\circ}$ anticlockwise to repeat the process carried out at the beginning to capture the opposite end of the pole.

\subsubsection{Scan registration and output}

Point cloud processing was performed according to the methodology established on Artec Studio 12 [32] which consisted of several steps that would align and register the points, to ultimately generate a more readable $3 \mathrm{D}$ file, known as mesh .OBJ file [35]. Comparative analysis was carried out to define the values of these point cloud process parameters which are summarised in Table 4.

Table 4. Point cloud processing description

Point cloud process

Description

Fine Registration

Aligns captured frames within one session, considering geometry and texture acquired.

Global Registration

Converts local point coordinates, contained in the frames, to a global coordinate system.

Outliers removal

Removes small surfaces unconnected to the main surface. 
Fast Fusion

Mesh Simplification
Melts and solidifies the point cloud into a triangulated mesh. polygons.

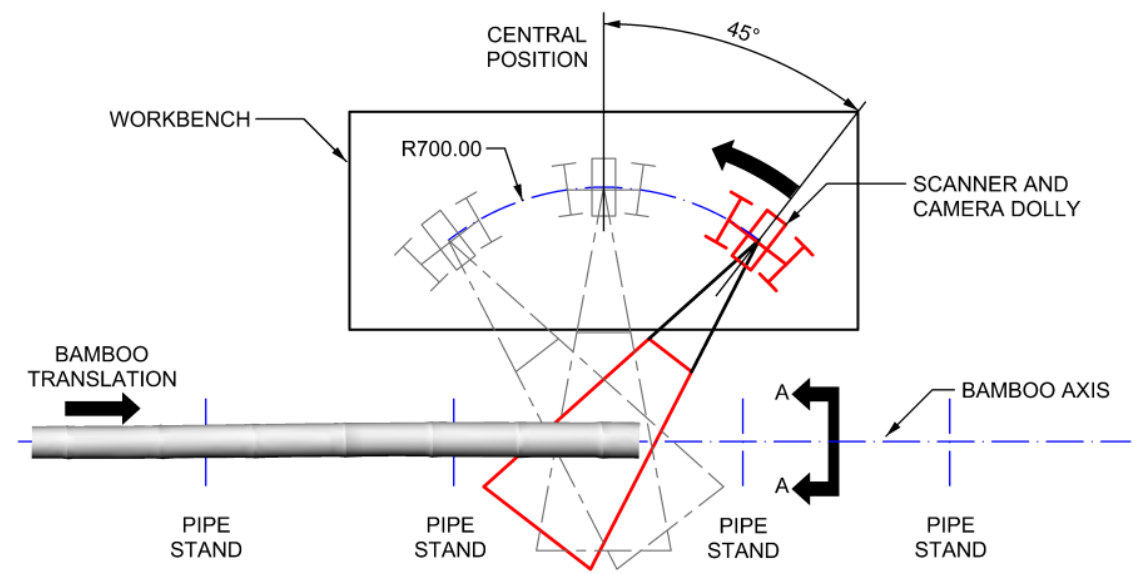

Figure 4. Bamboo scanning set-up

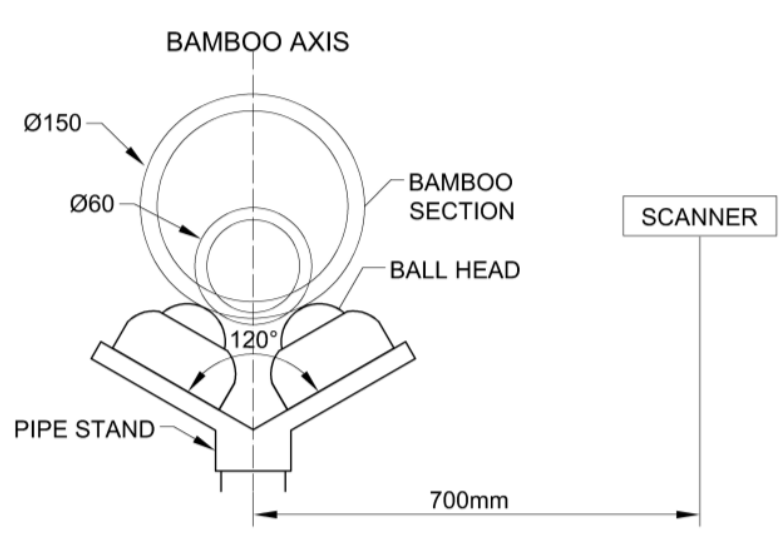

Figure 5. A-A section

\subsection{Digital and manual measurements}

\subsubsection{Digital measurements}

Key measurements were obtained from the mesh model in order for them to be validated against the corresponding manual measurements extracted from the physical poles. Due to occlusions, the inner surface of the bamboo was just acquired at the ends, however, according to the literature $[2,5,7,15]$ it has been found through the studies of different species (Phyllostachys Edulis Riv., Bambusa Pervariabilis, Phyllostachys Pubescens, Dendrocalamus Giganteus) that the wall thickness of bamboo tends to decrease linearly from bottom to top. Therefore, the portion of the inner surfaces were linearly extrapolated to generate an inner surface for each individual mesh file.

The diameter and thickness were extracted according to ISO 22157-1-2004 [16] which appoints that measurements are defined as the average of

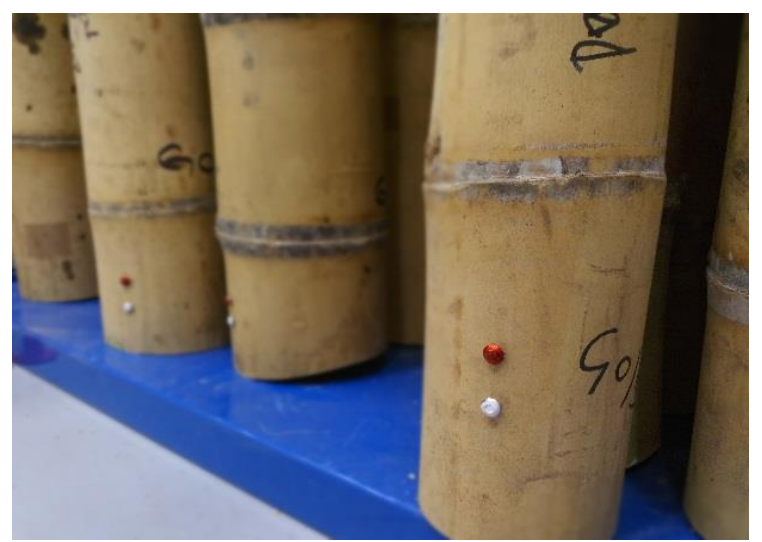

Figure 6. Scanning target references

measurements taken from each cross-section. Primarily, both outer and inner meshes were sectioned with a planar surface so that curves are fitted to the intersections (outer curve and inner curve). The area of each curve was then extracted, and the inner and outer equivalent diameters were calculated (Eq. 5a and 5b). An equivalent thickness was finally calculated as shown in Equation 6. Results of diameter and thickness were rounded to $1 \mathrm{~mm}$ and $0.1 \mathrm{~mm}$ following ISO 22157-1-2004 [16].

$$
\begin{gathered}
D_{o}=\sqrt{\frac{4 A_{o}}{\pi}} \\
D_{i}=\sqrt{\frac{4 A_{i}}{\pi}} \\
t_{e}=\frac{D_{o}-D_{i}}{2}
\end{gathered}
$$

Where $t_{e}$ is the equivalent thickness $(\mathrm{mm})$ of the section and $D_{0}$ and $D_{i}$ are the outer and inner equivalent 
diameters $(\mathrm{mm})$ calculated from the extracted outer and inner areas, $A_{o}$ and $A_{i}\left(\mathrm{~mm}^{2}\right)$ respectively. Areas and second moment of areas were then calculated from ISO 22157-1-2004 [16] (using $D_{0}$ as the average diameter and $t_{e}$ as the average thickness), and the values were rounded to $1 \mathrm{~mm}^{2}$ and $1 \mathrm{~mm}^{4}$ respectively. The length of the pole was taken as the linear distance between two scanning reference points located at each end of the bamboo. The virtual reading was rounded to $10 \mathrm{~mm}$, according to ISO 22157-1-2004 [16].

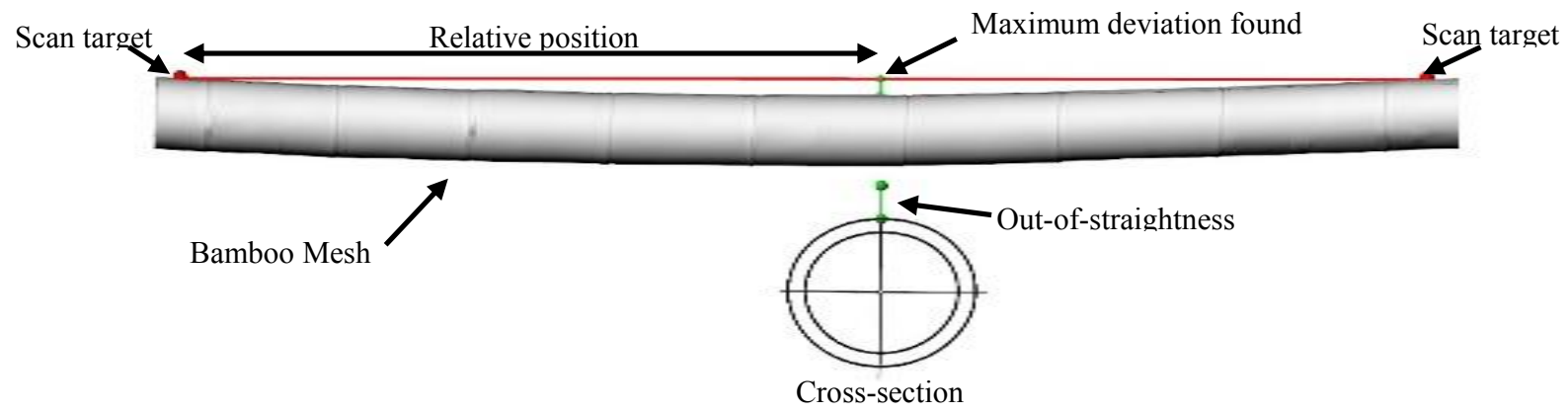

Figure 7. Virtual measurement of out-of-straightness

The out-of-straightness was measured from the virtual model as the deviation of the bamboo's surface from a straight line. As shown in Figure 7, the straight line was defined as a linear distance between two scanning reference points. The distance between the line and the surface was then evaluated along the line, until the maximum value was found. Out-ofstraightness and relative position were recorded. The plane where the distance is measured passes through the axis of the bamboo (Figure 7). Out-of-straightness is not a measurement defined by ISO 22157-1-2004 [16], however readings were rounded to $0.1 \mathrm{~mm}$.

\subsubsection{Manual measurements}

Manual measurements were taken from bamboo poles as a benchmark to compare against the results of the proposed digital workflow. These measurements followed the guidelines in ISO 22157-1-2004 [16] as shown in Figure 8 and were taken after cutting the bamboo at different sections along its length, rounded to $1 \mathrm{~mm}$ for diameter and $0.1 \mathrm{~mm}$ for thickness. Based on these measurements, average section properties were also calculated based on ISO 22157-1-2004 [16]:

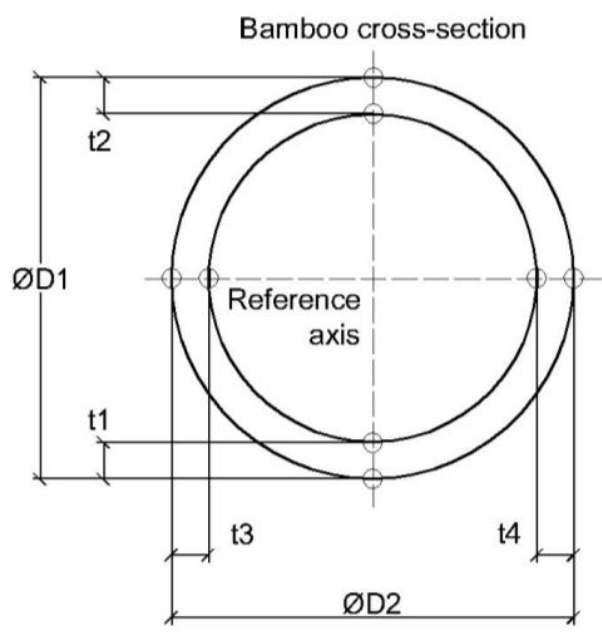

Figure 8. Measurements taken based on [36]

Cross sectional area:

$$
A_{c}=\left[\frac{\pi}{4}\right]\left[D^{2}-(D-2 t)^{2}\right]
$$

Second moment of area:

$$
I_{c}=\left[\frac{\pi}{64}\right]\left[D^{4}-(D-2 t)^{4}\right]
$$

Where $\mathrm{D}$ and $\mathrm{t}$ are the average diameter and thickness respectively measured in the cross-section shown in Figure 8. The total length was measured based on the scanning target references positioned at the bottom and top ends of the pole, consistent with the digital measurement. Readings were rounded to $10 \mathrm{~mm}$, according to ISO 22157-1-2004 [16].

The out-of-straightness was also measured with the aid of the scanning target references. As this parameter is not described in ISO 22157-1-2004 [16], a similar procedure to that followed in the digital process was manually applied. A thread was tied from target to target and the distance between the thread and 
bamboo's surface was recorded at the relative position distance where the out-of-straightness was found to be maximum, according to the digital model (Figure 7). The recorded measurement was rounded to a tolerance of $0.1 \mathrm{~mm}$.

\section{Validation and discussion}

The validation of the digital mesh model was carried out comparing its key dimensions against those in the real object. The results of the achieved accuracy of the selected parameters for each bamboo specie are shown in Table 5.

The digital workflow was successfully applied in all 3 species, finding that the developed process allows the digitisation of a great range of diameters, bamboo colours and shapes that differed among the species selected. The duration of the workflow (scanning and post-processing) and file sizes were in agreement with those estimated in Table 3. Moreover, the calculated scan overlapping provided enough information to generate accurate models.

Table 5. Summary of results for the different geometric parameters

\begin{tabular}{lccc} 
& & \multicolumn{2}{c}{ Accuracy (mm) } \\
\cline { 3 - 4 } $\begin{array}{l}\text { Geometric } \\
\text { parameter }\end{array}$ & Total Samples & Average & Std. Dev. \\
\hline Diameter & 105 & 0.65 & 0.78 \\
Thickness & 105 & 0.43 & 0.43 \\
Length & 15 & 3.05 & 2.47 \\
Out-of-straightness & 15 & 0.50 & 0.57
\end{tabular}

Table 6. Accuracy results for the compared section properties

\begin{tabular}{lccc} 
Geometric parameter & Total Samples & Average & Std. Dev. \\
\hline Area & 105 & $4 \%$ & $2 \%$ \\
Second moment of area & 105 & $3 \%$ & $3 \%$
\end{tabular}

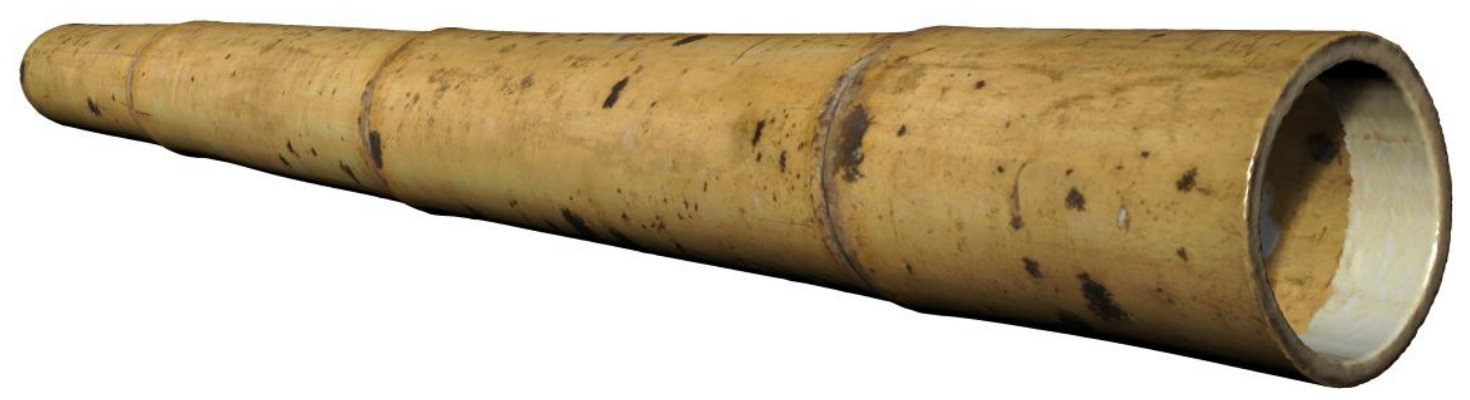

Figure 9. Virtual model of Guadua bamboo

A total of 25 bamboos were manually and digitally measured, from which ten belonged toOldhamii, ten to Guadua and five to Moso species. Including trials, a total of 500 additional linear meters of bamboo from three different species were scanned and processed showing the efficiency of the proposed workflow. These models form the basis of a wider database that allows further geometric analyses of the different imperfections in bamboo culms and therefore, consider these imperfections in their structural and mechanical behaviour (Figure 9).

As shown in Table 5, diameter and thickness (which were measured together on the same section) had a similar average accuracy of $0.65 \mathrm{~mm}$ and 0.43 $\mathrm{mm}$ respectively. These values were in accordance with the scanner specifications, indicating that the bamboo section extracted from the mesh model represented the true surface with a high level of accuracy. The average accuracy for the pole length was $3.05 \mathrm{~mm}$ over a total average length of $3.5 \mathrm{~m}$ which is considered a very good index of accuracy when compared with the standard tolerances for shapes and sizes applied to wood. According to BS EN 336:2013 [37] an allowance of -3 to $5 \mathrm{~mm}$ of tolerance is accepted on dimensions over $300 \mathrm{~mm}$.

One of the most important parameters that can be accurately extracted using the bamboo virtual model obtained from the scanning methodology is the out-of- 
straightness. As Ghavami \& Moreira [5] pointed out, the out-of-straightness is a deviation on bamboo's main axis that diminish the loading capacity of the element in conjunction with negative effects in stresses and deformations. Previous methods to measure this parameter $[5,8]$ have demonstrated to have good agreement with experimental tests, however, the manual methods proposed would be difficult to implement due to their complexity and time-consuming nature. The average accuracy obtained for this parameter was $0.5 \mathrm{~mm}$ which increases the level of confidence in the digital methodology applied. The digital method to calculate the maximum out-ofstraightness for each pole demonstrated that even the most complex dimensions can be easily extracted, and even automated, to obtain high accuracy results. When measuring the out-of-straightness by hand however, the task was complicated and time consuming, diminishing the confidence in the method applied.

In addition to the manual measurements, the section properties of the real and virtual models were also compared (Table 6). The average value for area and second moment of area were $4 \%$ and $3 \%$ respectively. Both parameters were calculated based on the average diameter and thickness of each section, and therefore, these results confirm the accuracy of results obtained in the validation of dimensions.

In summary, dimensional parameters and cross section properties showed a good agreement between the digital model and the physical pole. As explained previously, reliability and repeatability of the manual measurements are always compromised when applied to irregular objects, hence, a more systematic method should be applied to meet the quality assurance requirements in the building industry. The workflow presented in this research shows a remarkable improvement in bamboo geometry acquisition, it considerably reduces the measuring-process time and diminishes uncertainties on the results. Moreover, it allows the user to have a better understanding of the geometric variability and its implications in the project where the scanned poles are to be used.

\section{Conclusion}

This research presents the details of a digital workflow to quantify the inherent geometric variability of bamboo poles that can form part of a new quality assurance process for this natural structural element. This workflow relies on the use of a mid-range, commercially available structured-light $3 \mathrm{D}$ scanner to generate a polygon mesh model of the poles.

During the development of the methodology, the key parameters and scanning methodology were analysed and discussed to optimise the process both in terms of accuracy and efficiency. The driving parameter for the scanning process was found to be the total number of frames captured during the scan session, as the file size and point cloud processing time are directly dependent on it. The methodology was also designed to ensure that each pole is captured in a single scan to add to the efficiency of the process.

To validate the model, manual and digital measurements were taken from 25 bamboo culms of three different species. Hand measure methods have been developed for years, yet there are different factors that can still affect the final accuracy, however, the workflow presented in this research shows a remarkable improvement in bamboo geometry acquisition, reduction of the measuring-process time and diminishment of uncertainties in the measurements recorded. An overall accuracy under $1 \mathrm{~mm}$ was verified for diameter, thickness, and out-of-straightness. For the overall length, an average accuracy of $0.87 \mathrm{~mm} / \mathrm{m}$ was achieved. The process developed in this work is therefore considered as an accurate 3D workflow to reverse engineer bamboo culms, as the overall validated accuracies are well below the $10 \mathrm{~mm}$ accuracy threshold suggested for prototyping or reverse engineering applications [38].

This work demonstrates the benefits of adopting a non-destructive, reverse-engineering approach to quantify the geometric properties of bamboo compared to traditional tools and methods. Overall, this research shows the potential of digital technologies to support the adoption of this natural material allowing for the reassessment of traditional design workflows and providing an opportunity for natural bamboo culms to compete with industrialised materials.

\section{Acknowledgements}

The work presented in this paper was supported by the UK Engineering and Physical Sciences Research Council (EPSRC) (Grant Nos: EP/M017702/1 \& EP/P510890/1). The authors would like to thank Dr Gerardo Oliva-Salinas, Dr David Trujillo and Dr Li Haitao for enabling the procurement of the bamboo samples used in this study as well as Dr Chuhee Lee for her support during the implementation of the 3D scanning workflow.

\section{References}

1. Grosser D and Liese W (1971) On the anatomy of asian bamboos, with special reference to their vascular bundles. Wood Science and Technology 5: 290-312

2. Amada S, Ichikawa Y, Munekata T, Nagase Y and Shimizu H (1997) Fiber texture and mechanical graded structure of bamboo. Composites Part B 28B: $13-20$

3. Liese W (1987) Research on bamboo. Wood Science and Technology 21: 189-209

4. Hunter IR (2002) Bamboo - solution to problems. Journal of Bamboo and Rattan, 1(2): 101-107

5. Ghavami K and Moreira LE (2002) The influence of initial imperfection on the buckling of bamboo 
columns. Asian journal of Civil Engineering (Building and Housing) 3(3 \& 4): 1-16

6. Janssen JJA (2000) Designing and building with bamboo. International Network for Bamboo and Rattan, Beijing China

7. Chung KF and Yu WK (2002) Mechanical properties of structural bamboo for bamboo scaffoldings. Engineering Structures 24(4): 429-442

8. Richard MJ (2013) Assessing the performance of structural bamboo components. PhD thesis at the University of Pittsburg, United States of America

9. Sharma B, Harries KA and Ghavami K (2013) Methods of determining transverse mechanical properties of full-culm bamboo. Construction and Building Materials 38: 627-637

10. Trujillo D, Jangra S and Gibson JM (2017) Flexural properties as a basis for bamboo strength grading. Proceedings of the Institution of Civil Engineers, Structure and Buildings 170(SB4):284-294

11. Yu WK, Chung KF and Chan SL (2003) Column buckling of structural bamboo. Engineering Structures 25: 755-768

12. Arce, O (1993) Fundamentals of the design of bamboo structures. $\mathrm{PhD}$ thesis at the Technical University of Eindhoven, The Netherlands

13. Janssen JJA (1981) Bamboo in building structures. $\mathrm{PhD}$ thesis at the Technical University of Eindhoven, The Netherlands

14. Albermani F, Goh GY and Chan SL (2007) Lightweight bamboo double layer grid system. Engineering Structures 29: 1499-1506

15. Paraskeva TS, Grigoropoulos G and Dimitrakopoulos EG (2017) Design and experimental verification of easily constructible bamboo footbridges for rural areas. Engineering Structures 143: 540-548

16. ISO (International Organization for Standardization) (2004) ISO 22157-1:2004: Bamboo Determination of physical and mechanical properties - part 1: requirements. ISO, Geneva, Switzerland

17. Nurmadina, Nugroho N and Bahtiar ET (2017) Structural grading of Gigantochloa apus bamboo based on its flexural properties. Construction and Building Materials 157: 1173-1189

18. Harries KA, Bumstead J, Richard M and Trujillo D (2017) Geometric and material effects on bamboo buckling behaviour. Proceedings of the Institution of Civil Engineers, Structure and Buildings 170(SB4):236-249

19. Lorenzo R, Lee C, Oliva-Salinas JG and OntiverosHernandez MJ (2017) BIM Bamboo: a digital design framework for bamboo culms. Proceedings of the Institution of Civil Engineers, Structure and Buildings 170(SB4): 295-302

20. Konecny G (1985) The international society of photogrammetry and remote sensing - 75 years old, or 75 years young. Ibid 51(7): 919-933

21. Miles J, Pitts M, Pagi H and Earl Graeme (2014) New applications of photogrammetry and reflectance transformation imaging to an Easter Island statue. Antiquity 88: 596-605
22. Nicolae C, Nocerino E, Menna F and Remondino F (2014) Photogrammetry applied to problematic artefacts. The International Archives of the Photogrammetry, Remote Sensing and Spatial Information Sciences, ISPRS Technical Commission V Symposium XL-5: 451-456

23. Martinez-Llario J, Coll E and Herraez J (2006) Three-dimensional scanner software using a video camera. Advances in Engineering Software 37: 484-489

24. Shih NJ, Wang HJ, Lin CY and Liau CY (2007) 3D scan for the digitall preservation of a historical temple in Taiwan. Advances in Engineering Software 38: 501-512

25. Yang WB, Chen MB and Yen YN (2011) An application of digital point cloud to historic architecture in digital archives. Advances in Engineering Software 42: 690-699

26. Fechteler P, Eisert P and Rurainsky Jurgen (2007) Fast and high resolution $3 \mathrm{D}$ face scanning. IEEE International Conference on Image Processing 3: $81-84$

27. Jeon JH, Jung ID, Kim JH, Kim HY and Kim WC (2015) Three-dimensional evaluation of the repeatability of scans of stone models and impressions using a blue LED scanner.Dental Materials Journal 34(5): 686-691

28. Boehler W and Marbs A (2004) 3D scanning and photogrammetry for heritage recording: a comparison. Proc. 12th Int. Conf. on Geoinformatics - Geospatial Information Research: Bridging the pacific and atlantic. University of Gävle, Sweden 291-298

29. Abdelhafiz A (2013) Laser scanner point cloud colouring algorithm applied on real site. Survey Review 45(332): 343-351

30. MacDonald L, de Almeida VM and Hess M (2016) Three-dimensional reconstruction of Roman coins from photogrametric image sets. Journal of Electronic Imaging 26(1): 011017-1 - 20

31. Gibson LJ, Ashby MF, Karam GN, Wegst U and Shercliff HR (1995). The mechanical properties of natural materials. II. Microstructures for mechanical efficiency. 450(1938): 141-162

32. Artec 3D (2018) See https://www.artec3d.com/ (accessed 23/04/2018)

33. Fofi D, Sliwa T and Voisin Y (2004) A comparative survay on invisible structured light. Proc. SPIE Machine Vision Applications in Industrial Inspection XII 5303: 90

34. Bernal C, de Agustina b, Marin MM and Camacho AM (2013) Performance evaluation of optical scanner based on blue LED structured light. Procedia Engineering 63: 591-598

35. ISO (International Organization for Standardization) (2004) ISO 22157-2:2004: Bamboo Determination of physical and mechanical properties - part 2: Laboratory manual. ISO, Geneva, Switzerland

36. Wikipedia (2018) Wavefront .obj file. See https://en.wikipedia.org/wiki/Wavefront_.obj_file (accessed 23/04/18) 
37. BS EN (British adoption of European Standard) (2013) BS EN 336:2013: Structural timber. Sizes, permitted deviations. British Standards, United Kingdom
38. Runne H, Niemeier W and Kern F (2001) Application of laser scanners to determine the geometry of building. Optical 3-D measurement techniques

IV:

$41-48$ 